\title{
1. Die hugenottische Herkunft
}

Wenn ein Mensch sich einer geistigen Tradition verpflichtet fühlt, so ist dies nicht nur auf sein eigenes Bemühen und seine bewußt unternommenen Studien zurückzuführen, sondern in der Regel in seinem Herkommen und in seiner Familientradition angelegt. Allerdings muß es keineswegs zur Übernahme vorgegebener geistiger Traditionen, es kann auch im Gegenteil zur Ablehnung und einer entsprechend bewußten Identifikation mit gegenteiligen Traditionen kommen. Klepper gehört zu denjenigen, die ihre Familientradition angenommen, sie auch in mancher Hinsicht als brauchbare Grundlage für das spätere Leben betrachtet, sie aber doch abgewandelt und weiterentwickelt haben $^{1}$. Da der hugenottische Einfluß auf unsere deutsche Geschichte im Sinne der oben skizzierten demokratischen Tradition von großer Bedeutung war und eine wichtige Verbindung zu Frankreich geschaffen hat, ist es von Interesse, am Beispiel Kleppers auf diese Zusammenhänge kurz zu verweisen.

Die Vorfahren Kleppers waren sowohl mütterlicher- wie väterlicherseits Hugenotten, ein Ursprung, mit dem Klepper sich ein Leben lang identifiziert hat ${ }^{2}$. Dabei spielte für ihn die Verwurzelung in der westeuropäischen sowie in der protestantisch-christlichen Tradition eine ebenso wichtige Rolle wie Patriotismus und Zivilcourage, die als Einheit, nicht als Gegensatz verstanden und gelebt wurden. Symbolisch für diese Haltung der Hugenotten war für Klepper der Familienspruch, der aus dem 2. Brief des Paulus an Timotheus stammt und sich auf eine den Hugenotten vertraute Situation bezieht, nämlich die Einrichtung und Leitung einer Gemeinde in einer feindlichen, zumindest fremden Umwelt: „Gott hat uns nicht gegeben den Geist der Furcht, sondern der Kraft und der Liebe und der Zucht. " ${ }^{3}$ Die Hugenotten hatten in langen Kämpfen gegen die französische Staatsmacht eine freie Religionsausübung zu verteidigen sowie Mut zum Ungehor$s^{4}{ }^{4}$ einzuüben gelernt. Im neuen Zufluchtsland, neben Brandenburg-Preußen besonders auch Hessen-Cassel, hatten sie Fleiß, Anpassungsfähigkeit, freies Unternehmertum und Patriotismus, im Sinne einer freiwilligen Identifizierung mit dem ,neuen ' Vaterland, entwickelt, alles Fähigkeiten, die in dem Familienspruch zum Ausdruck kommen. Da die hugenottischen Familien bis Mitte des 19. Jahrhunderts „eigentlich nur unter sich

\footnotetext{
${ }^{1}$ Nach seiner Rückkehr aus dem Exil trat Klepper dem einst von seinem Vater mitbegründeten Familienverband bei mit der Begründung, daß er „, die Idee, die Verbindung, zwischen den Angehörigen unserer Familie durch Erforschung ihrer Tradition zu unterbauen', für richtig hielt, ,denn das einer Familie Gemeinsame liegt in der Vergangenheit "“ (Familienverbandszeitschrift, 9. Folge, Weinheim 1957, S. 10, KNL).

2 Otto Klepper an Mary Beth, Mexiko, 12.6. 1946. „Familiensinn“ sei in seiner Familie „traditionell", und er finde "es ungemein interessant, sich selbst genealogisch zu betrachten".

${ }^{3}$ 2. Thimotheus, 1,7. Klepper zitierte den Familien- und zugleich Taufspruch seines Sohnes Hermann Otto in einem Brief an seine Schwiegertochter Mary Beth zu der Geburt ihres ersten Kindes, Brief vom 27. 12. 1946 aus Mexiko.

${ }^{4}$ Klepper ebenda: "Courage civil, was den Deutschen so häufig fehlt".
} 
selbst geheiratet haben "5, ist die von ihnen überlieferte Tradition besonders wirksam geblieben.

Kleppers Mutter, Karoline Emilie Ottilie, war eine geborene Klingender, Tochter des Oberlandesgerichtsrats Otto Gustav Klingender ${ }^{6}$. Die Klingenders leiten sich laut Klepper von dem französischen König Henri IV. (1589-1610) ab. Einer seiner Nachkommen, ein Herzog von Lothringen, sei nach der Aufhebung des Edikts von Nantes ermordet worden, weil er sich geweigert habe, katholisch zu werden. Seine Witwe sei mit zwei kleinen Kindern nach Köln geflohen', und der Sohn sei dann später an den Hof des Landgrafen von Hessen-Cassel gekommen und Offizier geworden. Er führe als Stammvater seiner Familie noch das bourbonische Wappen, allerdings statt der zwei gekreuzten Klingen zwei abgebrochene Klingen, eben ,Klingenenden', im Schilde.

Der Ururgroßvater von Klepper, Johannes Friedrich Klingender, war, nachdem er fröhliche und abwechslungsreiche Lehr- und Wanderjahre in vielen Ländern Europas verlebt hatte, „Pfarrer der französischen Gemeinde und Inspektor der französisch-reformierten Gemeinden in Kurhessen" ${ }^{8}$. Er lebte in Kassel, heiratete die Tochter des berühmten Malers Tischbein des Älteren und zeugte mit ihr acht Kinder, von denen eines Wilhelm Elias, der Urgroßvater von Klepper, war. Dieser hatte in der Tradition seines eigenen Großvaters, der in der ersten Hälfte des 18. Jahrhunderts „aus Patriotismus vier Domänengüter gepachtet hatte“, Landwirtschaft, „Ökonomie und Tierarztkunde“ studiert". Da er sehr früh, sechs Wochen nach der Geburt seines vierten Kindes, starb, mußte sich die Witwe Klingender selbst helfen, was ihr mit Geschick gelang, so daß die Kinder - unter ihnen Kleppers Großvater - gesichert und in einer von Gläubigkeit, Liebe, Zucht und Pflichtbewußtsein geprägten Atmosphäre aufwachsen konnten.

Der Großvater Kleppers, Otto Gustav Klingender, scheint das Vorbild Kleppers gewesen zu sein; er studierte, wie später Klepper, Jura, trat in eine Verbindung ein und focht um "der Leibesübungen willen“, auch hatte er ein waches historisch-politisches Interesse. Er heiratete relativ spät ${ }^{10}$, und entsprechend kam seine Tochter Ottilie, die Mutter Kleppers, erst 1859 zur Welt. Sie hatte keine sehr kräftige Konstitution, schaffte es zwar, die Ausbildung als Lehrerin abzuschließen, hat ihren Beruf aber nur kurzzeitig ausgeübt.

1887 heirateten Kleppers Eltern: Ottilie Klingender und Konrad Martin Klepper, einziger Sohn des Kaufmanns und Tuchfabrikanten Karl Friedrich Klepper aus Kassel11. Dieser war 1858 in Kassel geboren worden und dort auch zur Schule gegangen, und zwar in die berühmte „Hohenzollernklasse“, zusammen mit dem späteren Kaiser Wil-

\footnotetext{
${ }^{5}$ Klepper an Mary Beth, Mexiko, 21.8. 1944.

6 "Selbstbiographie meines lieben Vaters, des am 11. Oktober 1887 zu Kassel entschlafenen Oberlandesgerichtsrats Otto Gustav Klingender“, Kleppers Großvater (1817-1887).

7 Eine Gedenktafel an dem Hause, in dem sie gewohnt habe, zeuge noch davon. Otto Klepper an Mary Beth, Mexiko, 21. 8. 1944.

8 Siehe die amüsant geschriebene „Selbstbiographie meines Urgroßvaters, des Professors der Weltweisheit und Inspektor der französischen Kolonien von Kurhessen, Pfarrer Johannes, Friedrich Klingender zu Kassel“, Kleppers Ururgroßvater (1747-1829). Sie beginnt mit dem Satz: „Frohund Leichtsinn sind die ständigen Begleiter auf meiner Lebensbahn gewesen."

${ }^{9}$ Biogr. 1817-1887.

${ }^{10}$ Ebenda, S. 22., im Juli 1852, Emilie, geb. Noll.

${ }^{11}$ Näheres über die Herkunft der Kleppers vgl. Thalmann, S. 12 f. Jochen Klepper war ein Vetter Otto Kleppers.
} 
helm II. Klepper war „in allen Klassen Primus-omnium“12. Später studierte er Jura, und wie in einem Nachruf des Familienblattes zu lesen ist, war Martin Klepper „ein allzeit liebenswürdiger, mitfühlender und hilfsbereiter Mensch mit heiterem Gemüt. Ihn adelte eine unbedingte Wahrheitsliebe, ein großes Pflichtgefühl und eine Treue und Zuverlässigkeit im Kleinsten wie im Großen. Er war ein geborener Gelehrter, von besonderem Wissensdrang und besaß einen auffallend scharfen Verstand und Herzensgüte, [...] eine unermüdliche Arbeitsfreude, verbunden mit Gewissenhaftigkeit und Gründlichkeit, [...]. und er war ein tiefgläubiger Christ." 13

Im Dreikaiserjahr, zwei Monate nach dem Tode Kaiser Friedrich III., am 17. August 1888, wurde Otto Klepper in Brotterode ${ }^{14}$, einem „Marktflecken im Kreise Schmalkalden", auf den Höhen des Thüringerwaldes ${ }^{15}$ geboren; ein Brotteroder also, es sind „Leute von Mut und Entschlossenheit“, wie es heißt ${ }^{16}$. Da Otto der einzige Sohn von Martin Klepper war, hatte dieser einen sehr großen Einfluß auf seine Entwicklung. Eine überlieferte Anekdote illustriert die Art der Prägung. Wenn Otto etwas ausgefressen hatte, hielt der Vater, der Oberlandesgerichtsrat, eine Gerichtsverhandlung ab, wobei er den Richter spielte. Seine Mutter hatte die Anklage zu vertreten, endete aber stets als Verteidiger ihres Sohnes und bat um Gnade. Einen solchen Vater zu haben, bedeutete sicher nicht nur Hilfe, sondern auch Belastung, eine hoch gehängte Meßlatte, die Versagensängste auslösen oder zu Ehrgeiz und Geltungsdrang führen konnte. Wie weit Otto an die von seinem Vater gesetzten Maßstäbe heranreichte, wird sich erweisen, Geltung hatten sie jedenfalls für ihn ein Leben lang. Besonders am christlichen Glauben hat er immer festgehalten, er bedeutete für ihn eine Kraftquelle ${ }^{17}$.

12 StAK, VbZ. Klep.: „Programm vom Schuljahr 1876/77 als Einladung zu der am 22. März 1877 stattfindenden öffentlichen Schulfeier des Geburtstages Sr. Majestät des Kaisers und Königs sowie zu der am 23. und 24. März 1877 abzuhaltenden öffentlichen Prüfung der Oberklassen des Gymnasiums" und Abiturientenliste des Königlichen Gymnasiums zu Cassel. Zusammen mit Prinz Wilhelm und dem Sohn des Staatsrats Wöhler erhielt M. Klepper eine Denkmünze als Auszeichnung für „Fleiß und sittliches Betragen“. Der Kaiser hat ihn später nach Wilhelmshöhe eingeladen und zu seinem Tode einen eigenhändig geschriebenen Beileidsbrief an die Witwe Klepper gesandt.

13 Adolf Klepper, Familienverbandszeitschrift, S. 126. Kleppers Vater hat rechtswissenschaftliche Arbeiten geschrieben, aber möglicherweise ist auch eine interessante historische, 200 Seiten umfassende Abhandlung von ihm, die sich „Die Kaisertragödie" nennt und genaueste Informationen zum Ablauf des Ersten Weltkrieges mit langen Zitaten von Groener u. a. enthält.

${ }^{14}$ Standesamt Brotterode Nr. 81/1888, Abschrift der Geburtsurkunde am 12. 1. 1955, Brotterode/ DDR, mit vollem Namen hieß er: Otto, Konrad, Wilhelm, Karl, Martin.

is Brotterode gehörte zum preußischen Regierungsbezirk Cassel, war Sitz eines Amtsgerichts, hatte 18902828 evangelische Einwohner, darunter 147 Reformierte, und war Standort für die Produktion von Stahl-, Eisen- und Drechslerwaren sowie Tabakanbau, außerdem war es ein Luftkurort, da es $600 \mathrm{~m}$ hoch über dem romantischen Trusental liegt. Am 10. 7. 1895 erlebte es eine furchtbare Feuersbrunst, bei der fast der ganze Ort niederbrannte. (Brockhaus, Berlin, 1895.) Im GSTAB, Rep. 84a, befinden sich „Generalienakten des Justizministeriums, betreffend den Wiederaufbau des niedergebrannten Marktfleckens und Amtsgerichts Brotterode, Kreis Schmalkalden", preußisches Justizministerium, Nr. 10745.

16 Kersten, Klepper, in: Aufbau, 24. 5. 1957. Kurt Kersten war in Kassel Nachbarskind von Klepper.

${ }_{17}$ Davon zeugen nicht nur seine Briefe an die Kinder, es wird auch bezeugt von Madeleine LandyDegon, die Klepper in Frankreich versteckt und sich viel mit ihm über Religion und Glauben unterhalten hat, sowie von Pfarrer Wolfgang Lehmann, der nach dem Kriege mit Klepper und der Wipog zusammengearbeitet hat. 


\section{Der Student und der Soldat}

War Familientradition und Elternhaus die eine Otto Klepper prägende Komponente, so war die zweite Schulausbildung, Studium und das Erleben des Ersten Weltkrieges. Er besuchte Schulen in Schmalkalden, Kassel ${ }^{18}$ und $\mathrm{Hamm}^{19}$, je nachdem, wohin sein Vater als Gerichts- und später Oberlandesgerichtsrat versetzt wurde. Vor 1905 war er auch noch im Pädagogium der evangelischen Brüdergemeinde in Niesky in der Oberlausitz. Seine Schulbildung war humanistisch und evangelisch-reformiert geprägt. $\mathrm{Da}$ „sein Vater den christlich-sozialen Gedanken Stoeckers ${ }^{20}$ und den Ideen Friedrich Naumanns ${ }^{21}$ sehr aufgeschlossen "22 gegenüberstand, interessierte sich auch der Sohn schon als Schüler für Politik und engagierte sich für die Christlich-Soziale Partei Adolf Stoeckers ${ }^{23}$.

Im Sommersemester 1908 begann Klepper sein Studium der Rechts- und Staatswissenschaften in Marburg ${ }^{24}$, dem traditionellen Studienort der Kleppers und Klingenders. Nachdem er zwischendurch noch in Berlin ${ }^{25}$, Münster ${ }^{26}$ und Göttingen ${ }^{27}$ studiert hatte, konnte er seine erste juristische Staatsprüfung gerade noch zu Beginn des Ersten Weltkrieges ablegen. Seine Ernennung zum Referendar erfolgte am 7. August 191428. Er hat relativ lange studiert, teilweise weil er sich politisch betätigte, teilweise weil er kein allzu eifriger Student war, teilweise auch, weil er einer studentischen Verbindung, dem Corps Hasso-Nassowia, beigetreten war. Er soll ein guter Fechter und ein guter Tänzer gewesen $\operatorname{sein}^{29}$. Auf die Frage, warum er sich duelliert habe, hat er seinem Sohn geantwortet, $\mathrm{da}$ ihn das gelehrt habe, körperlich mutig zu werden ${ }^{30}$.

${ }^{18}$ Er besuchte dort nur die Obersekunda Schuljahr 1905/6, Auskunft des Friedrichsgymnasiums und Schülerliste im Jahresbericht des Kgl. F.-Gymnasiums zu Cassel über das Schuljahr 1905/6, in: StAK.

${ }_{19} \mathrm{Im}$ preußischen Gymnasium Hamonese in Hamm hat er die beiden letzten Klassen des Gymnasiums besucht und 1908 das Abitur bestanden, Bestätigung durch das heutige Gymnasium in Hamm.

20 Adolf Stoecker (1835-1909) war bis 1890 Hofprediger in Berlin und gründete die Christlich-soziale Partei, mit deren Hilfe er die Arbeiterschaft im christlich monarchistisch-nationalen Sinne zu beeinflussen versuchte. Er war eine sehr umstrittene Figur, auch wegen seines antisemitischen Einflusses, vgl. Kampmann, S. $240 \mathrm{ff}$.

${ }^{21}$ Friedrich Naumann (1860-1919) kam über die Innere Mission zur Sozialpolitik und versuchte Stoeckers Ideen auf liberalerem Wege durchzuführen.

22 U. v. Pufendorf, in: O.W., Nr. 49, Mai/Juni 1957, S. 4 ff.

${ }^{23}$ Klepper an Georg Braun (Landgerichtspräsident in Göttingen), 19. 6. 1951, in: K/Kor. Vgl. auch Topf, S. 87: „Als Student hatte er sich den Christlich-Sozialen verschrieben, und zwar in der Stoeckerschen Richtung, die in Hessen-Kassel und Westfalen besonders florierte." (Alle folgenden Hinweise auf Topf beziehen sich, wenn kein anderer Titel vermerkt wird, auf sein Hauptwerk: „Die Grüne Front“.)

${ }^{24}$ HStA Marburg, Brief vom 12. 3. 1991 bestätigt Kleppers Immatrikulation vom 16. 5. 1908-10. 5. 1910 und 3. 11. 1910-5. 11. 1913.

25 AdHU Berlin: S-Semester 1910, Matrikelnummer 5716.

${ }^{26}$ S/W-Semester 1911/12, bestätigt durch Brief des AdU Münster vom 11. 12. 1990.

${ }^{27}$ GSTAB, Rep. 84a, Nr. 11150, Bl. 129 ff.: S-Semester 1912, sowie Bestätigung der anderen Daten.

28 Ebenda, Bl. 130. Prädikat: Gut.

${ }^{29}$ Auskunft des Sohnes: Er war ein beliebter und guter Tänzer, „because he was able to be a very charming person“. Georg Braun schrieb an Klepper, 16.6. 1951: Klepper „focht elegant und ,stand“ auffallend gut “. Kersten, Klepper: „Er ist ein guter Fechter gewesen und war stolz darauf.“

${ }^{30} \mathrm{Vgl}$. zur Tradition der schlagenden Verbindungen das aufschlußreiche Kapitel „Die satisfaktionsfähige Gesellschaft“ in Elias, Studien über die Deutschen, S. $61 \mathrm{ff}$. 
Der Eintritt in ein Corps war Tradition in der Familie, er hat sie unbefragt übernommen. Eventuelle Widersprüche zwischen seiner sehr christlichen und ethischen Normen verpflichteten Erziehung sowie seinem hugenottischen Erbe auf der einen und seiner Mitgliedschaft in einem konservativen, der ehemaligen Gruppe deutsch-nationaler Studentenschaft zugeneigten Corps ${ }^{31}$ auf der anderen Seite blieben zunächst verdeckt unter der Oberfläche. Doch drei Ereignisse, der Bruch mit dem Corps, die Erfahrung des Ersten Weltkrieges und der Kapp-Putsch, scheinen schließlich die Herausbildung einer klaren politischen Linie bewirkt zu haben.

Es ist wichtig, diese Ereignisse und die damit zusammenhängende politische Entwicklung Kleppers zu klären, denn sein für viele unverständliches Verhalten während seiner Studienzeit hat später häufiger dem Versuch gedient, ihn persönlich unglaubwürdig zu machen, um ihn als politischen Gegner auszuschalten. Klepper kommentierte dieses Vorgehen einmal in einer Rede als Finanzminister vor dem preußischen Landtag so: „Es ist eine der Geschmacklosigkeiten des politischen Kampfes der Gegenwart, daß man denjenigen, der ein öffentliches Amt übernimmt, wenn er sachliche Angriffsflächen nicht bietet, als in seiner persönlichen Ehrenhaftigkeit zweifelhaft darstellt. "32 Diese Antwort galt dem NSDAP-Abgeordneten Wilhelm Kube, der auf Kleppers Entfernung aus dem Corps Hasso-Nassowia angespielt hatte. Die Angelegenheit ist nicht mehr genau zu klären; weder wie weit er sich ursprünglich mit dem Geist des Corps identifiziert, noch was genau dazu geführt hat, daß ihm das Band des Corps aberkannt wurde. Es hat sich um eine Mensurensache gehandelt ${ }^{33}$, eine, wie Erwin Topf sagt, „ganz nichtige Begebenheit" ${ }^{34}$, die aber doch gravierende Folgen hatte. Georg Braun, Landgerichtspräsident in Göttingen und ehemaliger Corpsbruder von Klepper, schrieb nach dem Kriege zu diesen Vorgängen an Klepper folgendes: Nachdem Klepper verschwunden sei, habe „im Bund eine sehr gedrückte Stimmung“ geherrscht, und später habe er „von manchen Inaktiven ein sehr schlechtes Urteil über ihn [gebört], ein Urteil, das wir uns kritiklos alle zu eigen machten, ohne eigentlich zu wissen, was es bedeutete, das aber einer Art Diffamierung gleichkam, und das auch die Bemühungen einiger Alter Herren aus Kassel nicht zu korrigieren vermochten “35.

Vielleicht war dies für Klepper die erste einschneidende Erfahrung, ein outsider zu sein, eine Erfahrung, die er später noch häufig machen sollte. Topf deutet sie in diesem Sinne, indem er schreibt, es habe nun für Klepper nur noch eines gegeben: „Rehabilitieren, rehabilitieren! Dem Bund zeigen, daß man ohne ihn, gegen ihn sich durchsetzen kann! Das war Jahre hindurch die besondere Triebfeder für einen außergewöhnlichen und fast hemmungslosen Ehrgeiz. Fehlten ihm, dem Ausgestoßenen, die ,Beziehungen“ des Corps, so mußte er sich andere Verbindungen schaffen, gleichgültig in welchem Lager, denn die traditionellen gesellschaftlichen Bindungen bestanden für ihn ja nicht mehr im früheren Umfang. “36 Diese Deutung scheint übertrieben, zumal zwischen dem Bruch

${ }^{31}$ BAP, DNVP, 60 Vo2, 308, Bl. $2 \mathrm{ff}$.

32 PrLT, 283. Sitzung, 17. 3. 1932, Sp. 24725.

${ }^{33} \mathrm{G}$. Braun an Klepper, 16.6.51. In den Resten des noch bestehenden Corps-Archivs fand sich kein Hinweis auf Klepper.

${ }^{34}$ Topf, S. 88.

${ }^{35} \mathrm{G}$. Braun an Klepper, 16.6.51. In seinem Antwortbrief vom 19.6.51 erinnerte Klepper sich noch an die "unschönen Vorgänge von damals".

${ }^{36}$ Topf, S. 88. 
mit dem Corps, der wahrscheinlich 1910/11 in Marburg erfolgt war, zum einen sein Studium weiterging - ab 1912 sogar wieder in Marburg -, und zum anderen der Erste Weltkrieg mit seinen tiefgreifenden Veränderungen begann. Doch hat dieser Bruch sicher mit dazu beigetragen, daß Klepper eine scheinbar vorgezeichnete Bahn verließ und gezwungen war, sich neu zu orientieren.

$\mathrm{Zu}$ dieser Neuorientierung trugen erst recht die Erfahrungen im Ersten Weltkrieg bei. Er hatte sich - wie die meisten seiner Generation - freiwillig gemeldet. Seine Teilnahme am Krieg war von vornherein wegen Astigmatismus auf einem Auge eingeschränkt. Er wurde am 15. November 1916 eingezogen und diente bis zum 5. September 1917 als Meldereiter und dann wegen einer "schweren Verwundung“"37 als "Vaterländischer Hilfsdienstpflichtiger“. Als er als Leutnant der Reserve aus dem Kriegsdienst entlassen wurde, arbeitete er noch bis März 1919 „aus eigener Entschließung als Hilfsarbeiter bei der Reichsentschädigungskommission"38. Außerdem leistete er während des Krieges seinen juristischen Vorbereitungsdienst teils am Oberlandesgericht in Kassel, teils am Kammergericht in Berlin ab, so daß er im Dezember 1920 die Zweite juristische Staatsprüfung ablegen konnte ${ }^{39}$.

Die falsche Behauptung, Klepper habe der Deutschnationalen Volkspartei (DNVP) angehört ${ }^{40}$, läßt sich dadurch erklären, daß Teile der alten Stoecker-Partei in die nach dem Ersten Weltkrieg neu entstehende DNVP übergingen und daß diese zunächst „ein Sammelbecken von Konservativen, Freikonservativen, Deutsch-Völkischen, ChristlichSozialen und Reformern" war ${ }^{41}$. Als Referendar am Oberlandesgericht in Kassel ist Klepper während des Kapp-Putsches in Kassel anscheinend für die Aufrechterhaltung der Ordnung eingesetzt worden. Damals hat er den Jungdemokraten Ernst Lemmer festgenommen, was dieser auch in seinen Memoiren bestätigt und mit dem Satz kommentiert hat: „Oft haben wir uns in späteren Zeiten noch heiter über diese Episode unterhalten. " 42 Aus all diesen Vorfällen läßt sich der Gärungsprozeß eines jungen Mannes ablesen, der bei dem Versuch, in der scheinbar vorgezeichneten Richtung weiterzugehen, ständig aneckte und sich somit gezwungen sah, die Widersprüche dieser Umbruchzeit nach dem Ersten Weltkrieg zu verarbeiten und seinen eigenen Weg zu finden.

\footnotetext{
37 BAP, 62 DAF3, Nr. 17632, Bl. 107 f., Kurzbiographie, 1. 6. 1933 (Bl. 4052 f.).

${ }^{38}$ GSTAB, Rep. 84a, Nr. 11150, Bl. 130f. Dort traf er den späteren Freund Ludwig Aron.

${ }^{39}$ Ebenda, Bl. 131, Prädikat: ausreichend, ebenda: die genauen Daten seines Kriegs- und seines Referendardienstes.

40 Die Behauptung geht auf eine Anmerkung Topfs zurück, der sagt, Klepper habe sich im deutschnationalen Ortsverein Lichterfelde betätigt, sei aber bald "mit einem großen Krach“ ausgeschieden (Grüne Front, S. 88). Die Behauptung wurde vom DNVP-Abgeordneten Hans v. Rohr im PrLT, 264. Sitzung, 16. 12. 1931 (Sp. 23165), aufgegriffen und von Brüning in seinen Memoiren wiederholt (S. 481) und entsprechend in der wissenschaftlichen Literatur und in Lexikon-Artikeln zitiert. Weder in den Akten der DNVP im BAP noch im Berliner (O) Landesarchiv fand sich ein Hinweis auf Klepper. Klepper an Walter Hammer, 12. 10. 1954 und Klepper, Leserzuschrift für Das Ganze Deutschland, Freie Wochenzeitung, Nr. 10, 3. 3. 1951: „Ich habe früher keiner Partei angehört und bin nach wie vor parteilos“ (K/Kor.).

${ }^{41}$ Bredt, S. 27.

${ }^{42}$ Lemmer, S. 68.
} 


\section{Das Vorbild: Lorenz von Stein}

Wie stark Klepper durch sein Studium der Staats- und Rechtswissenschaft geprägt worden ist und wann er begonnen hat, sich mit der Gesellschaftslehre Lorenz von Steins zu beschäftigen, läßt sich nicht genau feststellen. Jedenfalls hat er Lorenz von Stein als seinen geistigen Vater bezeichnet und durch ihn die gesellschaftspolitische Linie gefunden, der er bis zum Ende seines Lebens treu geblieben ist ${ }^{43}$. Die Tatsache, daß Klepper seinem programmatischen Aufsatz „Vorfragen des Friedens" vom Herbst 1946 das folgende Stein-Zitat voranstellt, unterstreicht die Bedeutung, die Klepper Stein beigemessen hat: „Kein menschliches Auge reicht so weit, um sagen zu können, welches die letzte, vollendete und demnach im Widerspruch des unendlichen Geistes mit dem unendlichen Dasein erreichbare Ordnung der Gesellschaft ist. Allein die Beobachtung reicht dennoch weit genug, um das klar zeigen zu können, wodurch jede Ordnung hinter dieser höchsten Harmonie zurückbleibt" (1842). Was mag Klepper bewogen haben, gerade diesen Satz auszuwählen? Vielleicht glaubte er, mit ihm auszudrücken, was er selbst anstrebte, eine Vorstellung von einer vernünftigen gesellschaftlichen Ordnung zu entwickeln und pragmatisch zu versuchen, sich dieser anzunähern. Klepper war davon überzeugt, daß „Steins Konzept der Vermittlung von individueller Freiheit, gesellschaftlicher Abhängigkeit und staatlicher Autorität [...] eine realistische Staats- und Gesellschaftsphilosophie [sei], die das Zusammenspiel von ,Ideen' und ,Interessen' als Urheber der geschichtlichen Bewegung systematisch und empirisch angemessen abbildet" ${ }^{* 44}$. Wie Stein ging es Klepper darum, aus dem Studium der Staats- und Gesellschaftswissenschaften pragmatische Lehren für die Daseinsvorsorge als „eine Hilfe zur Selbsthilfe für jeden Einzelnen“ abzuleiten ${ }^{45}$. Es ging „um die Herstellung und Erhaltung der personalen Freiheit, vor allem der abhängigen Schichten im und durch den Staat " ${ }^{\text {46. }}$. „Selbstbestimmung der Persönlichkeit“, in dem Sinne, „daß der Mensch qualifiziert sei, selbst Herr seiner Lebensführung zu sein“ und „die Art [des] Zusammenlebens aus der Erkenntnis des Zweckmäßigen heraus selbst zu regeln“, ist nicht nur Recht, sondern auch Pflicht des Staatsbürgers. Beides habe sich nach Klepper aus der allmählichen Entfaltung des abendländischen individuellen Freiheitsbegriffs in der Geschichte entwickelt. Begonnen habe es mit der Erringung der "geistigen Freiheit" zur Zeit der Renaissance und Reformation. Während der Französischen Revolution sei dann die „staatsbürgerliche Freiheit“ erkämpft und im Laufe des 19. und 20. Jahrhunderts sei die Freiheit des einzelnen zur Grundlage der westlichen Demokratien geworden ${ }^{47}$.

Demnach sei das Ziel der Politik, die individuelle Freiheit der Menschen im Sinne der Präambel der amerikanischen Verfassung, "die Freiheit von der Not und von der Furcht“, tatsächlich zu realisieren, sie nicht nur in der Verfassung, sondern auch „im Gefüge der Ökonomie zu verankern“. Die daraus abzuleitende wichtigste Aufgabe der Po-

\footnotetext{
${ }^{43}$ In Heft Nr. 34 der O.W. (Nov./Dez. 1954) wurde ein Auszug aus Lorenz v. Steins „Der Sozialismus und Kommunismus des heutigen Frankreich. Ein Beitrag zur Zeitgeschichte" unter dem Titel „Das Prinzip der Gesellschaft" veröffentlicht. In Kleppers Nachlaß befindet sich eine ganze Mappe mit Auszügen aus Steins Werken sowie seine Geschichte der sozialen Bewegung.

${ }^{44}$ Koslowski, S. 178.

${ }^{45} \mathrm{v}$. Unruh, S. $458 \mathrm{f}$.

${ }^{46}$ Stein, Der Sozialismus, S. 447, zitiert bei v. Unruh, S. 460.

${ }^{47}$ Klepper, Gesellschaftskrise, Vortrag, 16. 3. 1948, S. 3 und Klepper, Bittere Wahrheit, S. 12.
} 
litik sei im Sinne von Lorenz von Stein, das Zusammenleben der Menschen dem Stande der sich verändernden Daseinsbedingungen anzupassen ${ }^{48}$. Das aber sei Aufgabe aller Staatsbürger. Es gehe nicht an, wie es "gemeinhin " geschehe, die unbefriedigende politische Situation „der Unzulänglichkeit der politischen Parteien und der parlamentarischen Körperschaften oder der Unverträglichkeit repräsentativer Persönlichkeiten zur Last“ zu legen, das sei „wohlfeil“ und verriete "nichts Besseres als den Versuch, aus der soeben zurückgewonnenen Selbstverantwortung unversehens zu entfliehen“. Wir bedürften der demokratischen Apparatur als eines notwendigen „Mittler[s] unseres eigenen Handelns und unseres Unterlassens“, sie bedeute "nicht weniger, aber auch nicht mehr“. „Die Ursachen“ für den Streit, der die nationalen Kräfte spaltet, statt sie in gemeinsamer Verantwortung zu vereinen, "liegen tiefer; nämlich in uns selbst“" ${ }^{\text {"49. Wenn }}$ Klepper diese Gedanken auch erst als älterer Mensch formuliert hat, als er nicht mehr handeln, sondern nur noch schreiben konnte, so sind sie doch schon Teil seines gesellschafts- und agrarpolitischen Konzeptes, das Grundlage seines politischen Handelns zur Zeit der Weimarer Republik war, und fanden damals schon entsprechenden Niederschlag in seinen politischen Artikeln und Reden.

Nicht nur Lorenz von Stein gehörte zu seinen geistigen Vätern, sondern auch die preußischen Reformer, vor allem der Freiherr vom Stein und Clausewitz, die er beide gerne zitierte. Auf dem Gebiet der Agrarwissenschaft war es vor allem Heinrich von Thünen, der ihm Anregungen gegeben hat.

Die Erkenntnis von der Selbstverantwortung des einzelnen lag aber auch in der Linie seiner hugenottischen Familientradition und war durch seine Erfahrungen als Student und Soldat bestätigt worden, es war eine frühe und eine zentrale Einsicht, die zur Maxime seines zukünftigen Handelns wurde.

Als seine berufliche Karriere im Juli 1921 begann $^{50}$, war er bereits seit dreieinhalb Jahren mit Gertrud Eickhoff, einer gebürtigen Norwegerin, verheiratet ${ }^{51}$ und hatte zwei Kinder. Die anderen beiden Kinder ${ }^{52}$ wurden kurze Zeit später geboren, so daß Klepper beim Start in sein Berufsleben bereits für eine große Familie verantwortlich war.

${ }^{48}$ Erste und Zweite Hattenheimer Resolution der Wipog vom Juni und August 1948.

${ }^{49}$ Klepper, Bittere Wahrheit, S. 7.

so GSTAB, Rep. 84a, Nr. 11150, Bl.131: Am 16.12. 1920 wurde er zum Gerichtsassessor im Bezirk des OLG-Kassel ernannt, ließ sich aber zur Wiederherstellung seiner Gesundheit bis zum 19. 3. 1921 beurlauben. Am 30. 6. 1921 trat er dann auf Antrag aus dem Justizdienst aus.

${ }^{51}$ Erna Gertrud Eickhoff, verw. Ziemer, * 25. 4. 1895 in Oslo/Norwegen, war eine Adoptivtochter von Prof. Dr. Hermann Eickhoff und Helene E., geb. Müller aus Hamm. Die Hochzeit fand am 18. 12.1917 statt (ebenda, Bl. 129).

52 Gisela (24. 9. 1918-20.3. 1925), Ingrid * 20. 2. 1921, Renate * 22. 9. 1922 und Martin Hermann Otto *31. 7. 1924. 